\section{EMPIRIC PROFILING OF PERIPHERAL T CELL RECALL RESPONSES TO TUMOR MUTANOMES VERSUS IN SILICO PREDICTIONS IN NSCLC PATIENTS UNDERGOING PEMBROLIZUMAB TREATMENT \pm CHEMOTHERAPY}

${ }^{1}$ Madison Milaszewski ${ }^{*},{ }^{1}$ James Loizeaux, ${ }^{1}$ Emily Tjon, ${ }^{1}$ Crystal Cabral, ${ }^{1}$ Tulin Dadali, ${ }^{1} J$ ustin Strickland, ${ }^{1} J a m i e$ Foti, ${ }^{1}$ Kevin Mancini, ${ }^{2}$ Kesi Michael, 'Louisa Dowal, ${ }^{1}$ Vijetha Vemulapalli, ${ }^{1}$ Alberto Visintin, ${ }^{1}$ Thomas Davis, 'Jessica Flechtner, ${ }^{2}$ Mark Awad. ${ }^{1}$ Genocea Biosciences, Cambridge, MA, USA; ${ }^{2}$ Dana-Farber Cancer Institute, Boston, MA, USA

Background Effective immune checkpoint blockade (ICB) treatment is dependent on T-cell recognition of patient-specific mutations (neoantigens). Empirical identification of neoantigens ex vivo has revealed shortcomings of in silico predictions. ${ }^{1}$ To better understand the impact of ICB treatment on $\mathrm{T}$ cell responses and differences between in silico and in vitro methods, neoantigen-specific $\mathrm{T}$ cell responses were evaluated in patients with non-small cell lung cancer undergoing first-line therapy with pembrolizumab \pm chemotherapy.

Methods Tumor and whole blood samples were collected from 14 patients prior to and after immunotherapy; seven each in monotherapy and combination therapy cohorts. The ex vivo ATLAS $^{\mathrm{TM}}$ platform was used to profile neoantigen-specific Tcell responses. Patient-specific tumor mutations identified by next-generation sequencing (NGS) were expressed individually as ATLAS clones, processed patient-specific autologous antigen presenting cells, and presented to their $\mathrm{T}$ cells in vitro. ATLAS-verified antigens were compared with epitope predictions made using algorithms.

Results On average, 150 (range 37-339) non-synonymous mutations were identified. Pre-treatment, ATLAS identified T cell responses to a median of $15 \%(9-25 \%)$ of mutations, with nearly equal proportions of neoantigens (8\%, 5-15\%) and Inhibigens ${ }^{\mathrm{TM}}$, targets of suppressive $\mathrm{T}$ cell responses $(8 \%$, $3-13 \%)$. The combination therapy cohort had more confirmed neoantigens $(46,20-103)$ than the monotherapy cohort $(7,6-$ 79). After treatment, the median ratio of CD4:CD8 $\mathrm{T}$ cells doubled in the monotherapy but not combination cohort (1.2 to 2.4 v. 1.6 to 1.3 ). Upon non-specific stimulation, $\mathrm{T}$ cells from patients on combination therapy expanded poorly relative to monotherapy ( 24 v. 65 -fold, $\mathrm{p}=0.014)$; no significant differences were observed pre-treatment $(22$ v. 18 -fold, $\mathrm{p}=$ 0.1578). Post-treatment, the median number of CD8 neoantigens increased in the combination therapy cohort (11 to 15) but in monotherapy were mostly unchanged (6 to 7). Across timepoints, $36 \%$ of ATLAS-identified responses overlapped. In silico analysis resulted in 1,895 predicted epitopes among 961 total mutations; among those, $30 \%$ were confirmed with ATLAS, although nearly half were Inhibigens, which could not be predicted. Moreover, 50\% of confirmed neoantigens were missed by in silico prediction.

Conclusions Monotherapy and combination therapy had differential effects on CD4:CD8 $\mathrm{T}$ cell ratios and their non-specific expansion. A greater proportion of neoantigens was identified than previously reported in studies employing in silico predictions prior to empirical verification. ${ }^{2}$ Overlap between confirmed antigens and in silico prediction was observed, but in silico prediction continued to have a large false negative rate and could not characterize Inhibigens.

Acknowledgements We would like to acknowledge and thank the patients and their families for participating in this study.

\section{REFERENCES}

1.. Lam H, McNeil LK, Starobinets H, DeVault VL, Cohen RB, Twardowski P, Johnson ML, Gillison ML, Stein MN, Vaishampayan UN, DeCillis AP, Foti JJ, Vemulapalli V Tjon E, Ferber K, DeOliveira DB, Broom W, Agnihotri P, Jaffee EM, Wong KK, Drake CG, Carroll PM, Davis TA, Flechtner JB. An empirical antigen selection method identifies neoantigens that either elicit broad antitumor T-cell responses or drive tumor growth. Cancer Discov 2021;11(3):696-713. doi: 10.1158/21598290.CD-20-0377. Epub 2021 January 27. PMID: 33504579.

2.. Rosenberg SA. Immersion in the search for effective cancer immunotherapies. Mol Med 27,63(2021). https://doi.org/10.1186/s10020-021-00321-3

http://dx.doi.org/10.1136/jitc-2021-SITC2021.248 